\title{
Etiology and Pathogenesis of Bloodstream Infections Associated with the Use of Long-Term Central Vascular Catheter (CVC) in Patients Who Undergone Gastrointestinal Surgery
}

\author{
Cristiane Silveira Brito ${ }^{1}$, Bruna Amâncio Gondim ${ }^{1}$, Augusto Diogo Filho ${ }^{2}$ and Paulo P. Gontijo Filho ${ }^{1}$ \\ ${ }^{1}$ Biomedical Sciences Institute, Uberlândia Federal University; ${ }^{2}$ Surgery Department of \\ Uberlândia Federal University Medical School; Uberlândia, MG, Brazil
}

\begin{abstract}
CVC is the main factor of risk of bloodstream infections. This study purpose was determining both etiology and pathogenesis of these infections in 80 patients who undergone gastrointestinal surgery and who worn long lasting CVC, in the institution HC-UFU. Cultures were made in nostril, skin of the insertion site, tip and catheter hub, in addition to hemoculture in those suspects of sepsis. The colonization incidence rate of the catheter tip was 12.5/ 1,000 catheter days and the CVC associate infection rate was 3.1/1,000 catheter days. Frequencies of skin, hub and catheter tip colonization were $13.8 \%, 8.9 \%$ and $13.3 \%$, respectively. Coagulase-negative Staphylococci were the most found microorganisms in nostril (74\%), skin (45.4\%) and hub (75\%) and Gram-negative bacilli (50\%), followed by $S$. aureus $(25 \%)$ the most common ones in catheter tip. Approximately $\mathbf{5 1 \%}$ of patients received antibiotics and most of them $(53.7 \%)$ had therapeutic purpose. The frequency of patients with clinical sepsis was $27.5 \%$. Three cases of bacteremia associated with the use of CVC were detected (3.8\%), with $S$. aureus in two of them and $K$. pneumoniae in the third one. There was not seen any association of skin and hub colonization with their presence in the catheter tip and in the blood of these patients, but $S$. aureus was recovered from nostril of those with sepsis by this pathogenic agent. A greater concern is suggested over preventive measures and control of these primary and secondary bloodstream, as well as catheter tip colonization.
\end{abstract}

Key Words: Nosocomial infection, central vascular catheter, bloodstream infections.

The use of central vascular catheter (CVC) was introduced into hospitals in the 1940's and become essential to the modern medical practice [1]. Although this intravascular device allows a rapid access into the bloodstream, its use has been associated to nosocomial bacteremias and candidemias, with significant rates of both morbidity and mortality, as well as high hospital costs [2]. They are used for intravenous administration of fluids, drugs, parenteral feeding, and hemodynamic monitoring of critical patients [3].

Mortality rate attributed to its use is of $12 \%-25 \%$, extending hospitalization by additional 10-40 days [4] which adds costs to the treatment about $\$ 33,000$ - 35,000/ patient [5].

The colonization source of the short-term ( $\leq 8$ days) catheter is the skin at the insertion site (75-90\%), followed by the catheter hub (10\%-50\%), bloodstream (3\%-10\% and over $50 \%$ in critical patients in Intensive Care Units - ICU) and infused material (2\%-3\%). On the other hand, for long-term CVCs ( $\geq 8$ days), the most frequent colonization source is the catheter cannon (66\%) followed by the skin with $26 \%$ [6].

According to the "National Nosocomial Infections Surveillance System” (NNIS) (2001) [7] the most commonly isolated pathogens in these infections, from January 1990 to May 1999. were: coagulase-negative Staphylococcus (37\%), followed by Staphylococcus aureus (13\%), Enterococcus sp. (13\%), Gram- negative bacilli (14\%) and Candida sp. (8\%).

Received on 16 July 2006; revised 5 December 2006.

Address for correspondence: Dr. Paulo P. Gontijo Filho. Uberlândia Federal University. Biomedical Sciences Institute, Campus Umuarama, Microbiology Laboratory. Av. Pará, 1720. Zip code: 38400-902. Uberlândia- MG, Brazil. E-mail: cristianebrito_bio@yahoo.com.br.

The Brazilian Journal of Infectious Diseases 2007;11(1):96-99. (c) 2007 by The Brazilian Journal of Infectious Diseases and Contexto Publishing. All rights reserved.
This study purpose was to evaluate etiology and pathogenesis of infections associated with CVCs in patients who undergone gastrointestinal surgery, as well as to find out the potential participation of nostril, skin at the insertion site, catheter hub, tip in the pathogenesis of these infections.

\section{Materials and Methods}

Patients Population

The study was carried out within the Clinic of Gastrointestinal Surgery of HC-UFU, 34 beds, of the Uberlândia University Medical School (HC-UFU), 503 beds, and included 80 patients who underwent surgery from October/2004-to December/2005. Patients were followed for three times/week from their entry into the study to their discharge or death. The ethical approval was obtained from the Ethics Committee of Uberlândia Federal University according to the Health Ministry demands. The written informed consent form was not asked because the procedure was considered standard practice of the institution care.

\section{Definitions}

Primary blood infection: bacteremia or candidemia without documentation of infection in known site [8].

Sepsis: needs one of the following signs and symptoms without any other documented cause: 1 ) fever $\geq 38^{\circ} \mathrm{C}$; 2) hypotension (systolic blood pressure $\leq 90 \mathrm{mmHg}$ ); and presence of the following conditions: not performed or negative hemoculture; clinical response to empirical antimicrobial therapy after catheter removal [8].

Catheter tip colonization: absence of infection signs at the catheter insertion site and microorganisms growth $\geq 10^{3}$ $\mathrm{UFC} / \mathrm{mL}$ (by quantitative culture) or $\geq 15 \mathrm{UFC} / \mathrm{mL}$ (by semiquantitative culture) [9]. 
Catheter associated infection: positive hemoculture with the same microorganism which is present on the catheter tip (by either quantitative or semi-quantitative culture) and clinical and microbiological absence of any other source of infection [9].

Microbiological Techniques

Skin of CVC Insertion Site. The collection of skin material was performed on the seventh day and after fourteen days of the catheter insertion, with a sterile saline pre-wet swab over a $20 \mathrm{~cm}^{2}$ area. The swab was put into a tube with $1 \mathrm{~mL}$ sterile saline and then stirred in a vortex; about $0.1 \mathrm{~mL}$ of the liquid was inoculated in Blood Agar plates which were incubated at $35^{\circ} \mathrm{C}$ for $24 \mathrm{~h}$. Cultures were considered positive if a growth of $\geq 200 \mathrm{UFC} / 20 \mathrm{~cm}^{2}$ was seen [10].

\section{CVCTip}

Catheters were removed under aseptic conditions, their tips were cut off with sterile scissors and the tips were transferred within sterile tubes to the Microbiology Laboratory. A quantitative culture was carried out according to the BRUNBUISSON and colleagues technique (1987) [11] which was considered positive when $\geq 10^{3} \mathrm{UFC} / \mathrm{mL}$ were present.

\section{Nostril- Catheter Hub}

Qualitative cultures of material collected from nostrils and catheter hub were performed. The swab was put into a tube containing sterile saline $1 \mathrm{~mL}$ and then stirred at vortex; about $0.1 \mathrm{~mL}$ of the liquid was inoculated in Blood Agar plates, McConkey, Salt Manitol, at $35^{\circ} \mathrm{C}$ for $24 \mathrm{~h}$ and Sabouraud Agar with chloramphenicol $16 \mu \mathrm{g} / \mathrm{mL}$ at $25^{\circ} \mathrm{C}$ for 5 days.

\section{Hemocultures}

Blood specimens were obtained from peripheral puncture. Hemocultures were performed by inoculating $5-10 \mathrm{~mL}$ of blood into a flask of the automatic commercial system Bactec/Alert ${ }^{\circledR}$ (Vitek System). Positive cultures were further sub-cultured in plates with McConkey Agar, Salt Manitol, Blood Agar, and Sabouraud Agar.

\section{Microrganims Identification}

The identification of samples was performed through traditional phenotype tests. Yeasts were characterized as Candida albicans by observing the formation of a germinative tube [12].

Test of Susceptibility to Antimicrobials

The test of diffusion in Agar was applied according to recommendations of "National Committee for Clinical Laboratory Standards" (NCCLS, 1997a) [13]. The sensitivity of positive samples from catheter tips and blood was analyzed by testing the following antimicrobials: ceftriaxone $(30 \mathrm{mcg})$, cefepime (30 $\mu \mathrm{g})$, cefoxitine (30 $\mu \mathrm{g})$, amoxicillin (30 mcg), amicacine $(30 \mu \mathrm{g})$, imipenem $(10 \mu \mathrm{g})$, aztreonam $(30 \mu \mathrm{g})$ and gentamicin $(10 \mu \mathrm{g})$ for isolated of Gram-negative bacilli and:

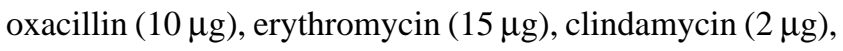
chloramphenicol (30 $\mu \mathrm{g})$, levofloxacin ( $5 \mathrm{mcg}$ ), sulfazotrin (25 $\mathrm{mcg}$ ) and rifampicin (5 mcg) for Staphylococci. Standard samples of Staphylococcus aureus ATCC 25923 and E. coli ATCC 25922 were used as controls.

\section{Statistical Analysis}

The statistical analysis of factors of risk for bloodstream infections was performed by applying the Fisher's exact test through the software Epi- Info 2000 (CDC-Atlanta). Statistical significance was defined as a p value $<0.05$.

\section{Results}

In total, 94 patients were investigated and 14 of them were excluded due to either non recovery of their catheter tips, or their removal to other units, or death. The average CVC lasting time was $10.7 \pm 4$ days/CVC with a 10 -day median. There was an average of 1.1 catheter/patient with 90 catheter tips being analyzed, from the 80 patients being investigated. Mean age of patients was 57 years with a range of 13-94 years, and a half of them were male. Most (80\%) of CVCs were inserted in the subclavian vein, and the other $20 \%$ in the jugular vein. The absence of occlusive bandage on the insertion site was seen for $18.9 \%$ of catheters.

Among the pathogenesis of infections associated to short and long-term CVCs, the most frequent sources of infection are insertion site skin and catheter hub, respectively. The catheter hub is mainly contaminated by the hands of health professionals with intraluminal dissemination up to the catheter tip [14]; or by translocation from gastrointestinal tract mucosa in very severe patients, usually in ICUs [15]. In this investigation, we found colonization of $8.9 \%$ of hubs but without any relationship with the recovered microorganism from both the catheter tip and the blood. In terms of pathogenesis, the only coincidence was the presence of two subjects with CVC-related bacteremia by $S$. aureus and this same organism was present in the nasal mucosa. Eleven (13.8\%) subjects were colonized $\left(\geq 200 \mathrm{UFC} / 20 \mathrm{~cm}^{2}\right)$ in the catheter insertion site, and the most frequent microorganisms (45.4\%) were SCN isolated. The nasal colonization reached $67.5 \%$ from which $74 \%$ by SCN, followed by S. aureus $20.4 \%$ (Table 1 ).

Positive results of analyzed tips reached $13.3 \%$, from which $50 \%$ by Gram-negative bacilli, $25 \%$ by $S$. aureus, $8.3 \%$ by SCN, $8.3 \%$ by C. albicans and $8.3 \%$ by Corynebacterium spp. Isolated of Gram-negative bacilli were identified as corresponding to the family of Enterobacteriaceae, species Klebsielleae (67\%) (Table 2). According to Pawar et al. (2004) [16] an increase occurs in the frequency of Gram-negative bacilli, which causes CVC-associated bloodstream infection in patients who undergone heart surgery.

Colonization rates of catheter tip, catheter-associated bloodstream infection, and clinical sepsis were $13.3 \%, 6.3 \%$ and $16.3 \%$, respectively. Central catheter-associated bloodstream infection rate was 3.1/1000 CVC days (Table 3). Data related to both prevalence and incidence of catheter- 
Table 1. Frequency of colonization in nasal mucosa, skin, hub and catheter tip, and bacteremia in 80 patients interned in the Clinic of Gastrointestinal Surgery of HC-UFU from October/2004 to December/ 2005

\begin{tabular}{|c|c|c|c|c|c|c|}
\hline \multirow[b]{2}{*}{ Microorganism } & \multicolumn{4}{|c|}{ Colonization (\%) } & \multirow{2}{*}{$\begin{array}{c}\text { Bacteremia/ Episodes } \\
\mathrm{N}=5(\%)\end{array}$} & \multirow{2}{*}{$\begin{array}{c}\text { CVC-associated } \\
\text { bacteremia } \mathrm{N}=3(\%)\end{array}$} \\
\hline & $\begin{array}{c}\text { Nostril } \\
N=54\end{array}$ & $\begin{array}{l}\text { Skin } \\
\mathrm{N}=11\end{array}$ & $\begin{array}{l}\text { Hub } \\
N=8\end{array}$ & $\underset{\mathbf{N}=12}{\text { Tip }}$ & & \\
\hline $\mathrm{SCN}^{*}$ & $40(74.0)$ & $5(45.4)$ & $6(75.0)$ & $1(8.3)$ & - & - \\
\hline S. aureus & $11(20.4)$ & $2(18.2)$ & $1(12.5)$ & $3(25.0)$ & $2(40.0)$ & $2(66.7)$ \\
\hline C. albicans & $2(3.7)$ & - & - & $1(8.3)$ & $2(40.0)$ & - \\
\hline $\mathrm{BGN}^{* *}$ & $1(1.9)$ & $2(18.2)$ & $1(12.5)$ & $6(50.0)$ & $1(20.0)$ & $1(33.3)$ \\
\hline Corynebacterium spp. & - & $2(18.2)$ & - & $1(8.3)$ & - & - \\
\hline
\end{tabular}

* Coagulase negative Sthaphylococcus. ${ }^{* *}$ Gram- negative bacilli.

Table 2. Gram-negative bacilli isolated from the catheter tip in patients interned in Clinic of Gastrointestinal Surgery of HCUFU, from October/2004 to December/2005

\begin{tabular}{lc}
\hline Microorganisms & $\begin{array}{c}\text { Total } \\
\mathbf{N}=\mathbf{6}(\mathbf{\%})\end{array}$ \\
\hline Escherichia coli & $2(33.3)$ \\
Enterobacter aerogenes & $2(33.3)$ \\
Citrobacter freundii & $2(33.3)$ \\
\hline
\end{tabular}

Table 3. Catheter tip colonization, bloodstream infections and catheter-associated in 80 patients interned in Clinic of Gastrointestinal Surgery of HC-UFU from October/2004 to December/ 2005

\begin{tabular}{lc}
\hline Rates & $\begin{array}{c}\text { Patients } \\
\mathbf{N}=\mathbf{8 0}\end{array}$ \\
\hline Catheter tip colonization (\%) & $12(13.3)$ \\
& 12.5 \\
Incidence of positive catheter tip/ & \\
1,000 catheter days & $5(6.3)$ \\
Incidence of bloodstream (\%) & $3(3.8)$ \\
CVC-associated bloodstream infection (\%) & 5.2 \\
Bloodstream infection 1000/ catheter days & $3(3.1)$ \\
CVC-associated bloodstream infection & \\
1,000/ catheter days & $22(27.5)$ \\
Clinical sepsis (\%) &
\end{tabular}

associated bloodstream infection, non antibiotic impregnated, ranged from $1.6 \%$ to $11.0 \%$ and from 2.3 to $16.8 / 1000$ catheter days in critical units, respectively [8].

In total, four subjects/five episodes of bacteremia were detected, from which three were associated to the CVC use and S. aureus (two episodes) and Klebsiella pneumoniae (one episode) were the etiologic agents for these infections. Currently, the main agent for primary bacteremia is SCN, with prevalence of S. epidermidis [17]. The participation of this microorganism is hardly defined due to the difficulty of separating from contamination infection [12]. In this study, it was not detected in cases of CVC-associated bacteremia, and it was only seen on $8.3 \%$ of catheter tips.

In two subjects with $S$. aureus as the etiologic agent of the CVC-associated infection, one of them presented this microorganism resistant to oxacillin, erythromycin, clindamycin, chloramphenicol, levofloxacin, sulfazotrin and rifampicin at catheter tip, nostril and blood, and another subject presented sensitivity to these drugs in all these sites. Samples of Klebsiella pneumoniae from the subject with bacteremia showed sensitivity to antimicrobial drugs.

We found that about $70 \%$ of Gram-negative bacilli isolated from catheter tips were resistant to one or more of the antibiotics tested. In terms of staphylococci samples, only one isolated acted as if it was a multi-resistant MRSA phenotype.

Results of cultures were negative in most of skin (80\%) and hubs (90\%) collections from the investigated subjects. This probably reflects the anti-sepsis care. About one third (30\%) of these subjects were using CVC for over 14 days, and amongst them a second collection was performed from the CVC insertion site, without qualitative differences compared to the first collection. The only change seen as a function of a greater internment time was the nasal colonization by $S$. aureus which was only detected at the second evaluation.

Among the predisposing factors of risk for CVCassociated bloodstream infections are: presence of multiluminal catheter, hemodialysis catheter, catheter-related thrombosis, anatomical site of insertion, catheterization lasting, difficulty of inserting the catheter, and hospital lasting before the insertion [18,19]. In our study, the stay of CVC $\geq 14$ days and the catheter tip colonization with a counting $\geq 10^{3} \mathrm{UFC} / \mathrm{mL}$, as well as the one of nasal mucosa by $S$. aureus were all significant ( $\mathrm{p} \leq 0.05)$.

Another major factor of risk related to nosocomial infection, especially those caused by resistant microorganisms, is the use of antibiotics [20]. In this series, it was not significant ( $>>0.05$ ) in CVC-associated bacteremia in spite of being prescribed to most of patients (51.3\%), from which about $54 \%$ with therapeutic purpose. 


\section{Conclusion}

In total, the frequency of patients with clinical sepsis was high (27.5\%), with prevalence of primary sepsis (59\%), but only three (23\%) were associated to CVCs. This data highlight the need of a greater concern on measures of control and prevention of CVC-associated bloodstream infections in hospital both for critical patients and non critical ones, especially in surgeries.

\section{References}

1. Worthington T., Elliot T.S.J. Diagnosis of central venous catheter related infection in adult patients. J Infect 2005; 51:267-80.

2. Garcia P.C., Paya E., Olivares R.C., et al. Diagnóstico de las infecciones asociadas a catéteres centrales. Rev Chil Infect 2003; $1: 41-50$.

3. O’ Grady N.P., Alexander M., Dellinger E.P., et al. Guidelines for the prevention of intravascular catheter-related infections. Clin Infect Dis 2002;35:1281-1307.

4. Safdar N., Fine J.P., Maki D.G. Meta-Analysis: Methods for diagnosing untravasacular device-related bloodstream infection. Ann Intern Med 2005;142:451-66.

5. Digiovine B., Chenoweth C., Watts C., Higgins M. The attributable mortality and costs of primary nosocomial bloodstream infections in intensive care unit. Am J Respir Crit Care Med 1999;160:976-81.

6. Sherertz R.J. Pathogenesis of Vascular Catheter Infection. In: Waldvogel F.A., Bisno A.L. Infections associated with indwelling medical devices. Washington: ASM Press. 2000;111-25.

7. National Nosocomial Infections Surveillance (NNIS) System Reporty, Data Summary from January 1992- June 2001, issued August 2001.Am J Infect Control 2001;29:404-21.

8. Eggimann P., Sax H., Pittet D. Catheter-related infections. Microbes Infect 2004;6:1033-42.
9. Eggimann P., Pittet D. Overview of catheter-related infections with special emphasis on prevention based on educational programs. Clin Microbiol Infect 2002;8:295-309.

10. Maki D.G., Ringer M., Lvarado C.J. Prospective randomized trial of povidine-iodine, alcohol e chlorexidine for prevention of infection associated with central venous and arterial catheters. Lancet 1991;338:339-43.

11. Brun-Buisson C., Abrouk F., Legrand P., et al. Diagnosis of central venous catheter- related sepsis: Critical level of quantitative tip cultures. Archs of Intern Med 1987;147:873-7.

12. Koneman E.W., Allen S.D., Janda W.M., et al. Diagnóstico Microbiológico: Texto e Atlas Colorido. Rio de Janeiro: MEDSI. 2001.

13. National Committee for Clinical Laboratory Standards (NCCLS). Performance Standards for Antimicrobial Disk Susceptibility Tests, 6Th ed. Approved M2-A6, 1997a.

14. Sherertz R.J., Ely E.W., Westbrook D.M., et al. Education of physiciansin-training can decrease the risk for vascular catheter infection. Ann Intern Med 2000;132:641-7.

15. Donlan R.M. Biofilms: microbial life on surfaces. Emerg Infec Dis 2003;8:881-90.

16. Falkenheuer G., Cornely O., Seifert H. Clinical management of catheter-related infections. Clin Microbiol Infect 2002;8:545-50.

17. Pawar M., Mehta Y., Kappor P., et al. Central venous catheterrelated blood stream infections: incidece, risk factors, outcome, and associated pathogens. J Cardiothorac Vasc Anesth 2004; 18 :304-8.

18. Reacher M.H., Shah A., Livermore M.D., et al. Bacteraemia and antibiotic resistence of its pathogens reported in England and Wales between 1990 and 1998:trend analysis. British Medical Journal 2000;320(22).

19. Viot M. Intravenous access: related problems in oncology. Intern. J. Antimicrob. Agents 2000;16:165-8.

20. Colombo A.L., Guimaraes T. Epidemiology of hematogenous infections due to Candida spp. Rev Soc Bras Med Trop 2003;26:599-607. 